ELECTRONIC RESEARCH ANNOUNCEMENTS OF THE AMERICAN MATHEMATICAL SOCIETY

Volume 10, Pages 68-77 (August 2, 2004)

S $1079-6762(04) 00131-3$

\title{
A NEW INEQUALITY FOR SUPERDIFFUSIONS AND ITS APPLICATIONS TO NONLINEAR DIFFERENTIAL EQUATIONS
}

\author{
E. B. DYNKIN
}

(Communicated by Mark Freidlin)

\begin{abstract}
Our motivation is the following problem: to describe all positive solutions of a semilinear elliptic equation $L u=u^{\alpha}$ with $\alpha>1$ in a bounded smooth domain $E \subset \mathbb{R}^{d}$. In 1998 Dynkin and Kuznetsov solved this problem for a class of solutions which they called $\sigma$-moderate. The question if all solutions belong to this class remained open. In 2002 Mselati proved that this is true for the equation $\Delta u=u^{2}$ in a domain of class $C^{4}$. His principal toolthe Brownian snake-is not applicable to the case $\alpha \neq 2$. In 2003 Dynkin and Kuznetsov modified most of Mselati's arguments by using superdiffusions instead of the snake. However a critical gap remained. A new inequality established in the present paper allows us to close this gap.
\end{abstract}

\section{INTRODUCTION}

1.1. Diffusions and superdiffusions. We denote by $\mathcal{M}(S)$ the set of all finite measures, and by $\mathcal{P}(S)$ the set of all probability measures on a measurable space $S$. $\mathcal{B}(E)$ stands for the set of all positive Borel functions on $E$. We use notation $\langle u, \mu\rangle$ for the integral of $u$ with respect to a measure $\mu$, and notation $P\{A, Y\}$ for the integral $\int_{A} Y d P$.

Let $L$ be an elliptic differential operator of the second order in $\mathbb{R}^{d}$. Under mild assumptions on the coefficients of $L$, there exists a continuous Markov process $\xi=$ $\left(\xi_{t}, \Pi_{x}\right)$ in $\mathbb{R}^{d}$ whose transition density is a fundamental solution of the parabolic equation $\partial u / \partial t=L u$. We call this process a diffusion. For every open set $D$ we denote by $\tau_{D}$ the first exit time of $\xi$ from $D$.

Let $\psi$ be a positive Borel function on $\mathbb{R}_{+}=[0, \infty)$. Suppose that to every open set $D$ and every $\mu \in \mathcal{M}\left(\mathbb{R}^{d}\right)$ there corresponds a random measure $\left(X_{D}, P_{\mu}\right)$ on $\mathbb{R}^{d}$ such that, for every $f \in \mathcal{B}\left(\mathbb{R}^{d}\right)$,

$$
P_{\mu} e^{-\left\langle f, X_{D}\right\rangle}=e^{-\langle u, \mu\rangle}
$$

where $u$ satisfies the equation

$$
u(x)+\Pi_{x} \int_{0}^{\tau_{D}} \psi\left[u\left(\xi_{t}\right)\right] d t=\Pi_{x} f\left(\xi_{\tau_{D}}\right) .
$$

Received by the editors April 23, 2004.

2000 Mathematics Subject Classification. Primary 60H30; Secondary 35J60, 60J60.

Key words and phrases. Positive solutions of semilinear elliptic PDEs, superdiffusions, conditional diffusions, $\mathbb{N}$-measures.

Partially supported by the National Science Foundation Grant DMS-0204237.

(C)2004 American Mathematical Society 
We call the family $X=\left(X_{D}, P_{\mu}\right)$ a superdiffusion. [Heuristically, we have here a model of a random evolution of the cloud of particles, and $X_{D}$ is a mass distribution on $\partial D$ if each particle is frozen at the first exit from $D$.]

The existence of a superdiffusion is proved for a convex class of positive convex functions which contains the functions

$$
\psi(u)=u^{\alpha}, \quad 0<\alpha \leq 2 .
$$

[See, e.g., Chapter 4 in Dy02.]

By restricting the family $\left(X_{D}, P_{\mu}\right)$ to $D \subset E$ and $\mu \in \mathcal{M}(E)$ we define a superdiffusion in an open set $E$.

A new tool - a family of measures $\mathbb{N}_{x}, x \in E$ (defined on the same space $\mathcal{O} O$ as measures $P_{\mu}$ ) - was introduced in DK04. Our inspiration was the role played by an analog of these measures in Le Gall's theory of the Brownian snake1

The range $\mathcal{R}_{E}$ of a superdiffusion in a domain $E$ is a minimal closed set which supports, $P_{x}$-a.s. and $\mathbb{N}_{x}$-a.s., an exit measure $X_{O}$ for an arbitrary open set $O \subset E$ and for every $x \in E$.

1.2. Stochastic boundary values of harmonic functions. We say that a function $h$ in $E$ is harmonic in $E$ if $L h=0$ in $E$ and we use the notation $\mathcal{H}(E)$ for the set of all positive harmonic functions. If $E$ is smooth 22 then there exists a 1-1 correspondence between $\mathcal{H}(E)$ and $\mathcal{M}(\partial E)$. The harmonic function $h_{\nu}$ corresponding to $\nu \in \mathcal{M}(\partial E)$ is given by the formula

$$
h_{\nu}(x)=\int_{\partial E} k_{E}(x, y) \nu(d y)
$$

where $k_{E}(x, y)$ is the Poisson kernel for $L$ in $E$. For every $\nu \in \mathcal{M}(\partial E)$, there exists a random variable $Z_{\nu}$ such that

$$
Z_{\nu}=\lim \left\langle h_{\nu}, X_{D_{n}}\right\rangle \quad P_{x} \text {-a.s. and } \mathbb{N}_{x} \text {-a.s. }
$$

for every $x \in E$ and for every sequence $D_{n}$ exhausting $E 3^{3}$ We call $Z_{\nu}$ the stochastic boundary value of $h_{\nu}$.

The energy function for $\nu \in \mathcal{M}(\partial E)$ is defined by the formula

$$
\mathcal{E}_{x}(\nu)=\Pi_{x} \int_{0}^{\tau_{E}} \psi\left[h_{\nu}\left(\xi_{t}\right)\right] d t .
$$

\subsection{Principal result.}

Theorem 1.1. Suppose that $D$ is a smooth open subset of a smooth domain E. If $\nu$ is a finite measure concentrated on $\partial D \cap \partial E$ and if $\mathcal{E}_{x}(\nu)<\infty$, then

$$
\mathbb{N}_{x}\left\{\mathcal{R}_{E} \subset D^{*}, Z_{\nu} \neq 0\right\} \geq C(\alpha)\left[\mathbb{N}_{x}\left\{\mathcal{R}_{E} \subset D^{*}, Z_{\nu}\right\}\right]^{\alpha /(\alpha-1)} \mathcal{E}_{x}(\nu)^{-1 /(\alpha-1)}
$$

where $C(\alpha)=(\alpha-1)^{-1} \Gamma(\alpha-1)$ !

We prove Theorem 1.1 in Section 5 after the necessary tools have been prepared in Sections 2-4.

\footnotetext{
${ }^{1}$ Definitions of $\mathbb{N}_{x}$ and other tools mentioned in the Introduction will be given in Section 2.

${ }^{2}$ We use the term smooth for open sets of class $C^{2, \lambda}$ unless another class is indicated explicitly.

${ }^{3}$ Domains $D_{n}$ exhaust $E$ if $\bar{D}_{n} \subset D_{n+1}$ and if the union of $D_{n}$ is equal to $E$.

${ }^{4}$ Here $\Gamma$ is Euler's gamma-function.
} 
1.4. Applications to differential equations. We denote by $\mathcal{U}(E)$ the set of all positive solutions of the equation

$$
L u=\psi(u) \quad \text { in } E .
$$

We say that an element $u$ of $\mathcal{U}(E)$ is moderate if $u \leq h$ for some $h \in \mathcal{H}(E)$. There exists a 1-1 correspondence between the set $\mathcal{U}_{1}(E)$ of all moderate solutions and a subset $\mathcal{H}_{1}(E)$ of $\mathcal{H}(E): h \in \mathcal{H}_{1}(E)$ is the minimal harmonic function dominating $u \in \mathcal{U}_{1}(E)$, and $u$ is the maximal solution dominated by $h$. We put $\nu \in \mathcal{N}_{1}^{E}$ if $h_{\nu} \in \mathcal{H}_{1}(E)$. We denote by $u_{\nu}$ the element of $\mathcal{U}_{1}(E)$ corresponding to $h_{\nu}$. These elements are related by the formula

$$
u_{\nu}(x)+\mathcal{E}_{x}(\nu)=h_{\nu}(x) .
$$

If $\mathcal{E}_{x}(\nu)<\infty$ for some $x \in E$, then $\nu \in \mathcal{N}_{1}^{E}$ 罒

To every closed subset $K$ of $\partial E$ there correspond two elements of $\mathcal{U}(E) 6$

$$
w_{K}(x)=-\log P_{x}\left\{\mathcal{R}_{E} \cap K=\emptyset\right\}=\mathbb{N}_{x}\left\{\mathcal{R}_{E} \cap K \neq \emptyset\right\}
$$

and

$$
u_{K}(x)=\sup u_{\nu}(x)
$$

where the supremum is taken over all $\nu \in \mathcal{N}_{1}^{E}$ concentrated on $K$.

We say that $u \in \mathcal{U}(E)$ is $\sigma$-moderate if there exist moderate solutions $u_{n}$ such that $u_{n} \uparrow u$. All solutions $u_{K}$ are $\sigma$-moderate.

Theorem 1.1 in combination with the results presented in Chapter 11, Section 7.1 of Dy02 and in Dy04a], Dy04c, [DK03, DK04, Ku04 makes it possible to prove the following two theorems 7

Theorem 1.2. If $E$ is a domain of class $C^{4}$ and if $L$ is the Laplacian $\Delta$, then

$$
u_{K}=w_{K} \quad \text { for all closed } K \subset \partial E \text {. }
$$

Theorem 1.3. Under the conditions of Theorem 1.2 all elements of $\mathcal{U}(E)$ are $\sigma$-moderate.

[Marcus and Véron proved in MV04 that the equation (1.12) can be established by a purely analytical method applicable to all $\alpha>1$.]

\section{TOOLS}

2.1. $h$-transform and conditional diffusion. Suppose $\xi$ is a diffusion in a domain $E$ with the transition function $p_{t}(x, d y)$, and let $h \in \mathcal{H}(E)$. Then

$$
p_{t}^{h}(x, d y)=\frac{1}{h(x)} p_{t}(x, d y) h(y)
$$

is the transition function of a continuous Markov process $\left(\xi_{t}, \hat{\Pi}_{x}^{h}\right)$ in $E$ called the $h$-transform of $\xi$. We prefer to deal with measures $\Pi_{x}^{h}=h(x) \hat{\Pi}_{x}^{h}$ which depend linearly on $h$. Put $\Pi_{x}^{\nu}=\Pi_{x}^{h_{\nu}}$ and $\hat{\Pi}_{x}^{y}=\hat{\Pi}_{x}^{\delta_{y}}$ where $\delta_{y}$ is the unit mass at a point $y$. The process $\left(\xi_{t}, \hat{\Pi}_{x}^{y}\right)$ can be interpreted as a diffusion starting from $x \in E$ and conditioned to exit from $E$ at $y$.

The following lemma is proved, for instance, in [Dy02, page 103:

\footnotetext{
${ }^{5}$ This follows, for instance, from Theorem 3.2 of Chapter 8 in Dy02].

${ }^{6} w_{K}$ can be characterized as the maximal element of $\mathcal{U}(E)$ vanishing on $\partial E \backslash K$.

${ }^{7}$ Proofs of these theorems are sketched in Dy04b]. The complete proofs are contained in the forthcoming book Dy04d.
} 
Lemma 2.1. For every stopping time $\tau$ and every pre- $\tau$ positive $Y$,

$$
\Pi_{x}^{h} Y 1_{\tau<\tau_{E}}=\Pi_{x} Y h\left(\xi_{\tau}\right) 1_{\tau<\tau_{E}} .
$$

2.2. Measures $\mathbb{N}_{x}$. Denote by $\mathcal{Z}_{x}$ the class of all functions of the form

$$
Z=\sum_{1}^{n}\left\langle f_{i}, X_{O_{i}}\right\rangle
$$

where $O_{1}, \ldots, O_{n}$ is a finite family of neighborhoods of $x$ and $f_{1}, \ldots, f_{n} \in \mathcal{B}\left(\mathbb{R}^{d}\right)$. By Theorem 1.1 in DK04, for every $x \in E$, there exists a unique measure $\mathbb{N}_{x}$ with the properties:

(i) For every $Z \in \mathcal{Z}_{x}$,

$$
\mathbb{N}_{x}\left(1-e^{-Z}\right)=-\log P_{x} e^{-Z} .
$$

(ii) If $\bar{\Omega}$ is the intersection of $\left\{X_{O}=0\right\}$ over all neighborhoods $O$ of $x$, then $\mathbb{N}_{x}(\bar{\Omega})=0$.

2.3. Stochastic boundary values and range. Suppose that $u \in \mathcal{B}(E)$. A random variable $Z_{u}$ is called a stochastic boundary value of $u$ [we write $Z_{u}=\operatorname{SBV}(u)$ ] if

$$
Z_{u}=\lim \left\langle u, X_{D_{n}}\right\rangle \quad P_{x^{-}} \text {-a.s. and } \quad \mathbb{N}_{x^{-}} \text {-a.s. }
$$

for every $x \in E$ and every sequence $D_{n}$ exhausting $E$.

For every $u \in \mathcal{B}\left(\mathbb{R}^{d}\right)$, we put

$$
V_{D}(u)(x)=-\log P_{x} e^{-\left\langle u, X_{D}\right\rangle} .
$$

Denote by $\mathcal{U}^{-}(E)$ the set of $u$ such that $V_{D}(u) \leq u$ for all $D \subset E$. This condition holds for all $u \geq 0$ such that $L u \leq \psi(u)$ in $E$. In particular, it holds for $u \in \mathcal{H}(E)$. Since $V_{D}\left(u_{1}+u_{2}\right) \leq V_{D}\left(u_{1}\right)+V_{D}\left(u_{2}\right)$ (Theorem 2.1 of Chapter 8 in Dy02]), the sum of two elements of $\mathcal{U}^{-}(E)$ belongs to $\mathcal{U}^{-}(E)$.

By Theorem 1.2 in [DK04], a stochastic boundary value $Z_{u}$ exist for every $u \in$ $\mathcal{U}^{-}(E)$ and

$$
\mathbb{N}_{x}\left(1-e^{-Z_{u}}\right)=-\log P_{x} e^{-Z_{u}} .
$$

Formula (1.5) means that $Z_{\nu}=\operatorname{SBV}\left(h_{\nu}\right)$. If $\nu \in \mathcal{N}_{1}^{E}$, then $Z_{\nu}$ is also $\operatorname{SBV}\left(u_{\nu}\right)$.

By Theorem 1.3 in DK04, for every domain $E$, there exists a random closed set $\mathcal{R}_{E}$ with the properties:

(a) For every open $O \subset E$ and every $x \in E$, the measure $X_{O}$ is concentrated, $P_{x}$-a.s. and $\mathbb{N}_{x}$-a.s., on $\mathcal{R}_{E}$.

(b) If (a) holds for a random closed set $F$, then, for every $x \in E, \mathcal{R} \subset F P_{x}$-a.s. and $\mathbb{N}_{x}$-a.s.

We call $\mathcal{R}_{E}$ the range of $X$ in $E$. We denote by $\mathcal{R}$ the range of $X$ in $\mathbb{R}^{d}$.

2.4. More relations between measures $P_{x}$ and $\mathbb{N}_{x}$. By Theorem 1.4 in DK04, for every $u \in \mathcal{U}^{-}(E)$ and every Borel set $\Gamma \subset \partial E$,

(2.7) $-\log P_{x}\left\{\mathcal{R}_{E} \cap \Gamma=\emptyset, e^{-Z_{u}}\right\}=\mathbb{N}_{x}\left\{\mathcal{R}_{E} \cap \Gamma \neq \emptyset\right\}+\mathbb{N}_{x}\left\{\mathcal{R}_{E} \cap \Gamma=\emptyset, 1-e^{-Z_{u}}\right\}$.

This function is the maximal element of $\mathcal{U}(E)$ dominated by $w_{\Gamma}+u$.

By taking $Z=0$, we get

$$
-\log P_{x}\left\{\mathcal{R}_{E} \cap \Gamma=\emptyset\right\}=\mathbb{N}_{x}\left\{\mathcal{R}_{E} \cap \Gamma \neq \emptyset\right\} .
$$


It follows from (2.7) and (2.8) that, if

$$
P_{x}\left\{\mathcal{R}_{E} \cap \Gamma=\emptyset\right\}>0,
$$

then

$$
\mathbb{N}_{x}\left\{\mathcal{R}_{E} \cap \Gamma=\emptyset, 1-e^{-Z}\right\}=-\log P_{x}\left\{e^{-Z} \mid \mathcal{R}_{E} \cap \Gamma=\emptyset\right\} .
$$

By applying (2.7) to $\lambda Z$ and passing to the limit as $\lambda \rightarrow+\infty$, we get

$$
-\log P_{x}\left\{\mathcal{R}_{E} \cap \Gamma=\emptyset, Z=0\right\}=\mathbb{N}_{x}\left\{\mathcal{R}_{E} \cap \Gamma \neq \emptyset\right\}+\mathbb{N}_{x}\left\{\mathcal{R}_{E} \cap \Gamma=\emptyset, Z \neq 0\right\} .
$$

By Proposition 1.1 in [DK04,

$$
\mathbb{N}_{x} Z_{\nu}=P_{x} Z_{\nu} \text { if } P_{x} Z_{\nu}<\infty
$$

On the other hand, for every $f \in \mathcal{B}(\bar{D})$,

$$
P_{x}\left\langle f, X_{D}\right\rangle=\Pi_{x} f\left(\xi_{\tau_{D}}\right)
$$

(see, e.g., Dy02, Chapter 4, Lemma 4.1). It follows from (2.5), (2.11), (2.12), Fatou's lemma and the mean value property of harmonic functions, that

$$
\mathbb{N}_{x} Z_{\nu}=P_{x} Z_{\nu} \leq h_{\nu}(x)<\infty \quad \text { for every } \nu \in \mathcal{M}(\partial E) .
$$

Proposition 2.1. Suppose $x \in D, \Lambda$ is a Borel subset of $\partial D$ and $\mathcal{A}=\{\mathcal{R} \cap \Lambda=\emptyset\}$. We have $P_{x} \mathcal{A}>0$ and, for all $Z^{\prime}, Z^{\prime \prime} \in \mathcal{Z}_{x}$,

$$
\begin{aligned}
& \mathbb{N}_{x}\left\{\mathcal{A},\left(e^{-Z^{\prime}}-e^{-Z^{\prime \prime}}\right)^{2}\right\} \\
& \quad=-2 \log P_{x}\left\{e^{-Z^{\prime}-Z^{\prime \prime}} \mid \mathcal{A}\right\}+\log P_{x}\left\{e^{-2 Z^{\prime}} \mid \mathcal{A}\right\}+\log P_{x}\left\{e^{-2 Z^{\prime \prime}} \mid \mathcal{A}\right\} .
\end{aligned}
$$

If $Z^{\prime}=Z^{\prime \prime} P_{x}$-a.s. on $\mathcal{A}$ and if $P_{x}\left\{\mathcal{A}, Z^{\prime}<\infty\right\}>0$, then $Z^{\prime}=Z^{\prime \prime} \mathbb{N}_{x}$-a.s. on $\mathcal{A}$.

Proof. First, $P_{x} \mathcal{A}>0$ because $P_{x} \mathcal{A}=e^{-w_{\Lambda}(x)}$. Next

$$
\left(e^{-Z^{\prime}}-e^{-Z^{\prime \prime}}\right)^{2}=2\left(1-e^{-Z^{\prime}-Z^{\prime \prime}}\right)-\left(1-e^{-2 Z^{\prime}}\right)-\left(1-e^{-2 Z^{\prime \prime}}\right) .
$$

Therefore (2.14) follows from (2.9). The second part of the proposition is an obvious implication of (2.14) .

2.5. Properties of superdiffusions. The following properties are often used in the theory of superdiffusions. [They are a part of the definition of branching exit Markov systems, and superdiffusions are a special case of such systems (see [Dy02, Chapters 3 and 4).]

2.5.A. (Markov property) If $Y \geq 0$ is measurable with respect to the $\sigma$-algebra generated by $X_{D^{\prime}}, D^{\prime} \subset D$ and $Z \geq 0$ is measurable with respect to the $\sigma$-algebra generated by $X_{D^{\prime \prime}}, D^{\prime \prime} \supset D$, then

$$
P_{\mu}(Y Z)=P_{\mu}\left(Y P_{X_{D}} Z\right) .
$$

2.5.B. If $\mu(E)=0$, then $P_{\mu}\left\{X_{E}=\mu\right\}=1$.

We use 2.5.A 2.5.B and Proposition 2.1 to prove the next proposition.

Proposition 2.2. Let $D \subset E$ be two open sets. Then, for every $x \in D, X_{D}$ and $X_{E}$ coincide $P_{x}$-a.s. and $\mathbb{N}_{x}$-a.s. on the set $\mathcal{A}=\left\{\mathcal{R}_{D} \subset D^{*}\right\}$.

[Note that

$$
D^{*}=\{x \in \bar{D}: d(x, \Lambda)>0\}
$$

where $\Lambda=\partial D \cap E$.] 


\section{Relations Between SUPERDiffusions AND CONDitional DifFusions IN TWO OPEN SETS}

3.1. Now we consider two bounded smooth open sets $D \subset E$. We denote by $\tilde{Z}_{\nu}$ the stochastic boundary value of $\tilde{h}_{\nu}(x)=\int_{\partial D} k_{D}(x, y) \nu(d y)$ in $D$; $\tilde{\Pi}_{x}^{y}$ refers to the diffusion in $D$ conditioned to exit at $y \in \partial D$.

Theorem 3.1. Put $\mathcal{A}=\left\{\mathcal{R}_{D} \subset D^{*}\right\}$. For every $x \in D$,

$$
\mathcal{R}_{E}=\mathcal{R}_{D} \quad P_{x} \text {-a.s. and } \quad \mathbb{N}_{x} \text {-a.s. }
$$

and

$$
Z_{\nu}=\tilde{Z}_{\nu} \quad P_{x} \text {-a.s. and } \mathbb{N}_{x} \text {-a.s. on } \mathcal{A}
$$

for all $\nu \in \mathcal{N}_{1}^{E}$ concentrated on $\partial D \cap \partial E$.

Proof. $1^{\circ}$. First, we prove (3.1). Clearly, $\mathcal{R}_{D} \subset \mathcal{R}_{E} P_{x}$-a.s. and $\mathbb{N}_{x}$-a.s. for all $x \in D$. We get (3.1) if we show that, if $O$ is an open subset of $E$, then, for every $x \in D, X_{O}=X_{O \cap D} P_{x}$-a.s. on $\mathcal{A}$ and, for every $x \in O \cap D, X_{O}=X_{O \cap D} \mathbb{N}_{x}$-a.s. on $\mathcal{A}$. For $x \in O \cap D$ this follows from Proposition 2.2 applied to $O \cap D \subset O$ because $\left\{\mathcal{R}_{D} \subset D^{*}\right\} \subset\left\{\mathcal{R}_{O \cap D} \subset(O \cap D)^{*}\right\}$. For $x \in D \backslash O, P_{x}\left\{X_{O}=X_{D \cap O}=\delta_{x}\right\}=1$ $2^{\circ}$. Put

$$
D_{m}^{*}=\{x \in \bar{D}: d(x, E \backslash D)>1 / m\} .
$$

To prove (3.2), it is sufficient to prove that it holds on $\mathcal{A}_{m}=\left\{\mathcal{R}_{D} \subset D_{m}^{*}\right\}$ for all sufficiently large $m$. First we prove that, for all $x \in D$,

$$
Z_{\nu}=\tilde{Z}_{\nu} \quad P_{x} \text {-a.s. on } \mathcal{A}_{m} \text {. }
$$

We get (3.4) by proving that both $Z_{\nu}$ and $\tilde{Z}_{\nu}$ coincide $P_{x}$-a.s. on $\mathcal{A}_{m}$ with the stochastic boundary value $Z^{*}$ of $h_{\nu}$ in $D$.

Let

$$
E_{n}=\{x \in E: d(x, \partial E)>1 / n\}, \quad D_{n}=\{x \in D: d(x, \partial D)>1 / n\} .
$$

If $n>m$, then

$$
\mathcal{A}_{m} \subset \mathcal{A}_{n} \subset\left\{\mathcal{R}_{D} \subset D_{n}^{*}\right\} \subset\left\{\mathcal{R}_{D_{n}} \subset D_{n}^{*}\right\}
$$

We apply Proposition 2.2 to $D_{n} \subset E_{n}$ and we get that, $P_{x^{-a}}$.s. on $\left\{\mathcal{R}_{D_{n}} \subset D_{n}^{*}\right\} \supset$ $\mathcal{A}_{m}, X_{D_{n}}=X_{E_{n}}$ for all $n>m$, which implies $Z^{*}=Z_{\nu}$.

$3^{\circ}$. Now we prove that

$$
Z^{*}=\tilde{Z}_{\nu} \quad P_{x} \text {-a.s. on } \mathcal{A}_{m} \text {. }
$$

Consider $h^{0}=h_{\nu}-\tilde{h}_{\nu}$ and $Z^{0}=Z_{\nu}^{*}-\tilde{Z}_{\nu}$. If $y \in \partial D \cap \partial E$, then

$$
k_{E}(x, y)=k_{D}(x, y)+\Pi_{x}\left\{\tau_{D}<\tau_{E}, k_{E}\left(\xi_{\tau_{D}}, y\right)\right\}
$$

Therefore

$$
h^{0}(x)=\Pi_{x}\left\{\xi_{\tau_{D}} \in \partial D \cap E, h_{\nu}\left(\xi_{\tau_{D}}\right)\right\} .
$$

This is a harmonic function in $D$. It vanishes on $\Gamma_{m}=\partial D \cap D_{m}^{*}=\partial E \cap D_{m}^{*}$.

We claim that, for every $\varepsilon>0$ and every $m, h^{0}<\varepsilon$ on $\Gamma_{m, n}=\partial E_{n} \cap D_{m}^{*}$ for all sufficiently large $n$. [If this is not true, then there exists a sequence $n_{i} \rightarrow \infty$ such that $z_{n_{i}} \in \Gamma_{m, n_{i}}$ and $h^{0}\left(z_{n_{i}}\right) \geq \varepsilon$. If $z$ is a limit point of $z_{n_{i}}$, then $z \in \Gamma_{m}$ and $h^{0}(z) \geq \varepsilon$.] 
E. B. DYNKIN

All measures $X_{D_{n}}$ are concentrated, $P_{x}$-a.s., on $\mathcal{R}_{D}$. Therefore $\mathcal{A}_{m}$ implies that they are concentrated, $P_{x}$-a.s., on $D_{m}^{*}$. Since $\Gamma_{m, n} \subset D_{m}^{*}$, we conclude that, for all sufficiently large $n,\left\langle h^{0}, X_{D_{n}}\right\rangle<\varepsilon\left\langle 1, X_{D_{n}}\right\rangle P_{x^{-}}$a.s. on $\mathcal{A}_{m}$. This implies (3.5)).

$4^{\circ}$. If $\nu \in \mathcal{M}(\partial E)$ and $Z_{\nu}=\operatorname{SBV}\left(h_{\nu}\right)$, then

$$
\mathbb{N}_{x} Z_{\nu}=P_{x} Z_{\nu} \leq h_{\nu}(x)<\infty .
$$

Note that $P_{x} \mathcal{A}>0$. It follows from (3.8) that $Z_{\nu}<\infty P_{x}$-a.s. and therefore $P_{x}\left\{\mathcal{A}, Z_{\nu}<\infty\right\}>0$. By Proposition 2.1], (3.2) follows from (3.4) .

3.2. We also need the following result (see Dy04a, Lemma 3.2).

Theorem 3.2. Suppose that $D \subset E$ are smooth open sets. Denote by $\tilde{\mathcal{F}}$ the $\sigma$ algebra in $\Omega$ generated by the sets $\left\{s<\tau_{D}, \xi_{s} \in B\right\}$ where $s \geq 0, B \in \mathcal{B}(E)$. We have

$$
\tilde{\Pi}_{x}^{y} Y=\Pi_{x}^{y}\left\{\tau_{D}=\tau_{E}, Y\right\}
$$

for all $x \in D, y \in \partial E \cap \partial D$ and for all $Y \in \tilde{\mathcal{F}}$.

Corollary 3.1. If

$$
F_{t}=\exp \left[-\int_{0}^{t} a\left(\xi_{s}\right) d s\right]
$$

where $a$ is a positive continuous function on $[0, \infty)$, then, for $y \in \partial D \cap \partial E$,

$$
\tilde{\Pi}_{x}^{y} F_{\tau_{D}}=\Pi_{x}^{y}\left\{\tau_{D}=\tau_{E}, F_{\tau_{E}}\right\} .
$$

Indeed, it is easy to see that $F_{\tilde{\tau}} \in \tilde{\mathcal{F}}$.

\section{Equations Connecting $P_{x}$ And $\mathbb{N}_{x}$ With $\Pi_{x}^{\nu}$}

4.1.

Theorem 4.1. Let $Z_{\nu}=\operatorname{SBV}\left(h_{\nu}\right), Z_{u}=\operatorname{SBV}(u)$ where $\nu \in \mathcal{N}_{1}^{E}$ and $u \in \mathcal{U}(E)$. Then

$$
P_{x} Z_{\nu} e^{-Z_{u}}=e^{-u(x)} \Pi_{x}^{\nu} e^{-\Phi(u)}
$$

and

$$
\mathbb{N}_{x} Z_{\nu} e^{-Z_{u}}=\Pi_{x}^{\nu} e^{-\Phi(u)}
$$

where

$$
\Phi(u)=\int_{0}^{\tau_{E}} \psi^{\prime}\left[u\left(\xi_{t}\right)\right] d t .
$$

Proof. Formula (4.1) follows from Theorem 3.1 in Chapter 9 of [Dy02]. To prove (4.2), we observe that, for every $\lambda \geq 0, \lambda Z_{\nu}+Z_{u}=\operatorname{SBV}(v)$ where $v=\lambda h_{\nu}+u \in$ $\mathcal{U}^{-}(E)$ and therefore, by (2.6),

$$
\mathbb{N}_{x}\left(1-e^{-\lambda Z_{\nu}-Z_{u}}\right)=-\log P_{x} e^{-\lambda Z_{\nu}-Z_{u}} .
$$

By taking the derivatives with respect to $\lambda$ at $\lambda=0$ we get

$$
\mathbb{N}_{x} Z_{\nu} e^{-Z_{u}}=P_{x} Z_{\nu} e^{-Z_{u}} / P_{x} e^{-Z_{u}} .
$$

By Theorem 1.1 of Chapter 9 in Dy02,

$$
P_{x} e^{-Z_{u}}=e^{-u(x)} \text {. }
$$

\footnotetext{
${ }^{8}$ The differentiation under the integral signs is justified by (2.13).
} 
Therefore (4.4) follows from (4.1), 4.5) and 4.6).

Theorem 4.2. Suppose that $D \subset E$ are bounded smooth open sets and $\Lambda, L, D^{*}$ are the sets introduced in Theorem 1.1. Let $\nu$ be a finite measure on $\partial D \cap \partial E$, $x \in E$ and $\mathcal{E}_{x}(\nu)<\infty$. Put

$$
\begin{aligned}
w_{\Lambda}(x) & =\mathbb{N}_{x}\left\{\mathcal{R}_{D} \cap \Lambda \neq \emptyset\right\}, \\
v_{s}(x) & =w_{\Lambda}(x)+\mathbb{N}_{x}\left\{\mathcal{R}_{D} \cap \Lambda=\emptyset, 1-e^{-s Z_{\nu}}\right\}
\end{aligned}
$$

for $x \in D$ and let $w_{\Lambda}(x)=v_{s}(x)=0$ for $x \in E \backslash D$. For every $x \in E$, we have

$$
\begin{gathered}
\mathbb{N}_{x}\left\{\mathcal{R}_{E} \subset D^{*}, Z_{\nu}\right\}=\Pi_{x}^{\nu}\left\{A, e^{-\Phi\left(w_{\Lambda}\right)}\right\}, \\
\mathbb{N}_{x}\left\{\mathcal{R}_{E} \subset D^{*}, Z_{\nu} \neq 0\right\}=\int_{0}^{\infty} \Pi_{x}^{\nu}\left\{A, e^{-\Phi\left(v_{s}\right)}\right\} d s
\end{gathered}
$$

where $\Phi$ is defined by (4.3) and

$$
A=\left\{\tau_{E}=\tau_{D}\right\}=\left\{\xi_{t} \in D \text { for all } t<\tau_{E}\right\} .
$$

Remark. Since $\mathcal{E}_{x}(\nu)<\infty, \nu$ belongs to $\mathcal{N}_{x}^{E}$ and to $\mathcal{N}_{x}^{D}$.

Proof. $1^{\circ}$. If $x \in E \backslash D$, then, $\mathbb{N}_{x}$-a.s., $\mathcal{R}_{E}$ is not a subset of $D^{*}$ because $\mathcal{R}_{E}$ contains supports of $X_{O}$ for all neighborhoods $O$ of $x$ and we can choose $O$ such that $\bar{O} \cap D^{*}=\emptyset$. On the other hand, $\Pi_{x}^{\nu}(A)=0$. Therefore (4.8) and (4.9) hold independently of values of $w_{\Lambda}$ and $v_{s}$.

$2^{\circ}$. Now we assume that $x \in D$. Put $\mathcal{A}=\left\{\mathcal{R}_{D} \subset D^{*}\right\}$. We claim that

$$
\mathcal{A}=\left\{\mathcal{R}_{E} \subset D^{*}\right\} \quad \mathbb{N}_{x} \text {-a.s. }
$$

Indeed, $\left\{\mathcal{R}_{E} \subset D^{*}\right\} \subset \mathcal{A}$ because $\mathcal{R}_{D} \subset \mathcal{R}_{E}$. By Theorem 3.1 $\mathcal{A} \subset\left\{\mathcal{R}_{D}=\mathcal{R}_{E}\right\}$ $\mathbb{N}_{x}$-a.s. Hence, $\mathcal{A} \subset\left\{\mathcal{R}_{E} \subset D^{*}\right\}$.

By Theorem 3.1, $\mathcal{R}_{D}=\mathcal{R}_{E}$ and $Z_{\nu}=\tilde{Z}_{\nu} \mathbb{N}_{x}$-a.s. on $\mathcal{A}$. Therefore

$$
\begin{aligned}
\mathbb{N}_{x}\left\{\mathcal{R}_{E} \subset D^{*}, Z_{\nu}\right\} & =\mathbb{N}_{x}\left\{\mathcal{A}, Z_{\nu}\right\}=\mathbb{N}_{x}\left\{\mathcal{A}, \tilde{Z}_{\nu}\right\}, \\
\mathbb{N}_{x}\left\{\mathcal{R}_{E} \subset D^{*}, Z_{\nu} e^{-s Z_{\nu}}\right\} & =\mathbb{N}_{x}\left\{\mathcal{A}, Z_{\nu} e^{-s Z_{\nu}}\right\}=\mathbb{N}_{x}\left\{\mathcal{A}, \tilde{Z}_{\nu} e^{-s \tilde{Z}_{\nu}}\right\} .
\end{aligned}
$$

Formula (4.7) defines two elements of $\mathcal{U}(D)$. The stochastic boundary value $Z_{\Lambda}$ of $w_{\Lambda}$ in $D$ is equal to $\infty 1_{\mathcal{A}^{c}}$ (Remark 1.2 on p. 133 in $[\overline{D y 02} \mid)$ and therefore

$$
e^{-Z_{\Lambda}}=1_{\mathcal{A}} .
$$

By (2.7) and (2.8), $v_{s}(x)=-\log P_{x}\left\{\mathcal{R}_{D} \cap \Lambda=\emptyset, e^{-s Z_{\nu}}\right\}$ and, by Remark 2.1 on p. 137 in Dy02, the stochastic boundary value $Z^{s}$ of $v_{s}$ in $D$ is equal to $Z_{\Lambda}+s \tilde{Z}_{\nu}$. Hence,

$$
e^{-Z^{s}}=1_{\mathcal{A}} e^{-s \tilde{Z}_{\nu}}
$$

By (4.11), (4.12) and (4.13),

$$
\mathbb{N}_{x}\left\{\mathcal{A}, Z_{\nu}\right\}=\mathbb{N}_{x}\left\{\tilde{Z}_{\nu} e^{-Z_{\Lambda}}\right\}
$$

and

$$
\mathbb{N}_{x}\left\{\mathcal{A}, Z_{\nu} e^{-s Z_{\nu}}\right\}=\mathbb{N}_{x}\left\{\tilde{Z}_{\nu} e^{-Z^{s}}\right\}
$$


E. B. DYNKIN

By applying formula (4.2) to $\tilde{Z}_{\nu}$ and to the restriction of $w_{\Lambda}$ to $D$, we conclude from (4.14) that

$$
\mathbb{N}_{x}\left\{\mathcal{A}, Z_{\nu}\right\}=\tilde{\Pi}_{x}^{\nu} \exp \left[-\int_{0}^{\tau_{D}} \psi^{\prime}\left[w_{\Lambda}\left(\xi_{s}\right)\right] d s\right]
$$

and, by Corollary 3.1,

$$
\mathbb{N}_{x}\left\{\mathcal{A}, Z_{\nu}\right\}=\Pi_{x}^{\nu}\left\{A, e^{-\Phi\left(w_{\Lambda}\right)}\right\} .
$$

Analogously, 4.2 applied to the restriction of $v_{s}$ to $D$, in combination with 4.15) and (3.11), yields

$$
\mathbb{N}_{x}\left\{\mathcal{A}, Z_{\nu} e^{-s Z_{\nu}}\right\}=\Pi_{x}^{\nu}\left\{A, e^{-\Phi\left(v_{s}\right)}\right\} .
$$

Formula (4.8) follows from (4.17) and formula (4.9) follows from (4.18) because

$$
\mathbb{N}_{x}\left\{\mathcal{A}, Z_{\nu} \neq 0\right\}=\lim _{t \rightarrow \infty} \mathbb{N}_{x}\left\{\mathcal{A}, 1-e^{-t Z_{\nu}}\right\}
$$

and

$$
1-e^{-t Z_{\nu}}=\int_{0}^{t} Z_{\nu} e^{-s Z_{\nu}} d s
$$

\section{Proof of Theorem 1.1}

We use the following two elementary inequalities:

5.A. For all $a, b \geq 0$ and $0<\beta<1$,

$$
(a+b)^{\beta} \leq a^{\beta}+b^{\beta} .
$$

Proof. It is sufficient to prove (5.1) for $a=1$. Put $f(t)=(1+t)^{\beta}-t^{\beta}$. Note that $f(0)=1$ and $f^{\prime}(t) \leq 0$ for $t>0$. Hence $f(t) \leq 1$ for $t \geq 0$.

5.B. For every finite measure $M$, every positive measurable function $Y$ and every $\beta>0$,

$$
M\left(Y^{-\beta}\right) \geq M(1)^{1+\beta}(M Y)^{-\beta}
$$

Indeed $f(y)=y^{-\beta}$ is a convex function on $\mathbb{R}_{+}$, and we get $5 . \mathrm{B}$ by applying Jensen's inequality to the probability measure $M / M(1)$.

Proof of Theorem 1.1. $1^{\circ}$. If $x \in E \backslash D$, then, $\mathbb{N}_{x}$-a.s., $\mathcal{R}_{E}$ is not a subset of $D^{*}$ (see the proof of Theorem 4.2). Hence, both sides of (1.7) vanish.

$2^{\circ}$. Suppose $x \in D$. By (2.6), $\mathbb{N}_{x}\left(1-e^{-s Z_{\nu}}\right)=u_{s \nu}$. Thus (4.7) implies $v_{s} \leq$ $w_{\Lambda}+u_{s \nu}$. Therefore, by 5.A $v_{s}^{\alpha-1} \leq w_{\Lambda}^{\alpha-1}+u_{s \nu}^{\alpha-1}$ and, since $u_{s \nu} \leq h_{s \nu}=s h_{\nu}$, $\Phi\left(v_{s}\right) \leq \Phi\left(w_{\Lambda}\right)+s^{\alpha-1} \Phi\left(h_{\nu}\right)$.

Put $\mathcal{A}=\left\{\mathcal{R}_{E} \subset D^{*}\right\}$. It follows from (4.9) that

$$
\mathbb{N}_{x}\left\{\mathcal{A}, Z_{\nu} \neq 0\right\} \geq \Pi_{x}^{\nu}\left\{A, \int_{0}^{\infty} e^{-\Phi\left(w_{\Lambda}\right)-s^{\alpha-1} \Phi\left(h_{\nu}\right)} d s\right\} .
$$

Note that $\int_{0}^{\infty} e^{-a s^{\beta}} d s=C a^{-1 / \beta}$ where $C=\int_{0}^{\infty} e^{-t^{\beta}} d t$. Therefore (5.2) implies

$$
\mathbb{N}_{x}\left\{\mathcal{A}, Z_{\nu} \neq 0\right\} \geq C \Pi_{x}^{\nu}\left\{A, e^{-\Phi\left(w_{\Lambda}\right)} \Phi\left(h_{\nu}\right)^{-1 /(\alpha-1)}\right\} .
$$


The right side in (5.3) is equal to $C M\left(Y^{-\beta}\right)$ where $\beta=1 /(\alpha-1), Y=\Phi\left(h_{\nu}\right)$ and $M$ is the measure with the density $1_{A} e^{-\Phi\left(w_{\Lambda}\right)}$ with respect to $\Pi_{x}^{\nu}$. We get from (5.3) and 5.B that

$$
\begin{aligned}
\mathbb{N}_{x}\left\{\mathcal{A}, Z_{\nu} \neq 0\right\} \geq & C M(1)^{1+\beta}(M Y)^{-\beta} \\
& =C\left[\Pi_{x}^{\nu}\left\{A, e^{-\Phi\left(w_{\Lambda}\right)}\right\}\right]^{\alpha /(\alpha-1)}\left[\Pi_{x}^{\nu}\left\{A, e^{-\Phi\left(w_{\Lambda}\right)} \Phi\left(h_{\nu}\right)\right\}\right]^{-1 /(\alpha-1)} .
\end{aligned}
$$

By (4.8), $\Pi_{x}^{\nu}\left\{A, e^{-\Phi\left(w_{\Lambda}\right)}\right\}=\mathbb{N}_{x}\left\{\mathcal{R}_{E} \subset D^{*}, Z_{\nu}\right\}$ and, since $\Pi_{x}^{\nu}\left\{A, e^{-\Phi\left(w_{\Lambda}\right)} \Phi\left(h_{\nu}\right)\right\} \leq$ $\Pi_{x}^{\nu} \Phi\left(h_{\nu}\right)$, we have

$$
\mathbb{N}_{x}\left\{\mathcal{A}, Z_{\nu} \neq 0\right\} \geq C\left[\mathbb{N}_{x}\left\{\mathcal{R}_{E} \subset D^{*}, Z_{\nu}\right\}\right]^{\alpha /(\alpha-1)}\left[\Pi_{x}^{\nu} \Phi\left(h_{\nu}\right)\right]^{-1 /(\alpha-1)} .
$$

$3^{\circ}$. By the definition of $h$-transform, for every $f \in \mathcal{B}(E)$ and every $h \in \mathcal{H}(E)$,

$$
\Pi_{x}^{h} \int_{0}^{\tau_{E}} f\left(\xi_{t}\right) d t=\int_{0}^{\infty} \Pi_{x}^{h}\left\{t<\tau_{E}, f\left(\xi_{t}\right)\right\} d t=\int_{0}^{\infty} \Pi_{x}\left\{t<\tau_{E}, f\left(\xi_{t}\right) h\left(\xi_{t}\right)\right\} d t .
$$

By taking $f=\alpha h_{\nu}^{\alpha-1}$ and $h=h_{\nu}$ we get

$$
\Pi_{x}^{\nu} \Phi\left(h_{\nu}\right)=\alpha \mathcal{E}_{x}(\nu) .
$$

Formula (1.7) follows from (5.4) and (5.5).

\section{REFERENCES}

[Dy91] E. B. Dynkin, A probabilistic approach to one class of nonlinear differential equations, Probab. Th. Rel. Fields 89 (1991), 89-115. MR 1109476 (92d:35090)

[Dy02] - Diffusions, superdiffusions and partial differential equations, American Mathematical Society, Providence, RI, 2002. MR 1883198 (2003c:60001)

[Dy04a] _ On upper bounds for positive solutions of semilinear equations, J. Functional Analysis 210 (2004), 73-100. MR 2051633

[Dy04b] Superdiffusions and positive solutions of nonlinear partial differential equations, Uspekhi Matem. Nauk 59 (2004), to appear.

[Dy04c] - Absolute continuity results for superdiffusions with applications to differential equations, C. R. Acad. Sc. Paris, Série I, 338 (2004), 605-610. MR 2056468

[Dy04d] Superdiffusions and positive solutions of nonlinear partial differential equations, American Mathematical Society, Providence, RI, 2004, to appear.

[DK03] E. B. Dynkin and S. E. Kuznetsov, Poisson capacities, Math. Research Letters 10 (2003), 85-95. MR 1960126 (2003k:31005)

[DK04] _, $\mathbb{N}$-measures for branching exit Markov systems and their applications to differential equations, Probab. Theory and Related Fields, to appear.

[Ku04] S. E. Kuznetsov, An upper bound for positive solutions of the equation $\Delta u=u^{\alpha}$, Amer. Math. Soc., Electronic Research Announcements, to appear.

[MV04] M. Marcus and L. Véron, Capacitary estimates of positive solutions of semilinear elliptic equations with absorbtion, J. European Math. Soc., to appear.

[Ms02] B. Mselati, Classification et représentation probabiliste des solutions positives de $\Delta u=$ $u^{2}$ dans un domaine, Thése de Doctorat de l'Université Paris 6, 2002.

[Ms04] B. Mselati, Classification and probabilistic representation of the positive solutions of a semilinear elliptic equation, Memoirs of the American Mathematical Society 168 (2004), no. 798 , to appear.

Department of Mathematics, Cornell University, Ithaca, Ny 14853

E-mail address: ebd1@cornell.edu 